\title{
El Papel Ambiental de Zamorano: Protección del Ambiente y la Biodiversidad
}

\author{
José Manuel Mora ${ }^{1}$ y Lucía I. López ${ }^{2}$
}

Resumen. Zamorano es una universidad dedicada a la formación de profesionales en las ciencias agrícolas. La responsabilidad social y la ambiental son partes integrales de dicha formación. Un ejemplo de esta responsabilidad es que uno de los departamentos de la universidad es el de Ambiente y Desarrollo que imparte la carrera de Ingeniería en Ambiente y Desarrollo. Durante sus 70 años de existencia, Zamorano ha ido incrementado su accionar en pro del ambiente. Ya en 1948, el Dr. Popenoe, primer director de Zamorano, proponía la creación de una reserva biológica para la protección del bosque nublado del cerro Uyuca. Dicha gestión ha culminado hoy en día con el área protegida de más alta efectividad de manejo en Honduras. La Reserva Biológica de Uyuca es proveedora de servicios ecosistémicos de gran valor en donde destaca el agua para nuestra comunidad. Zamorano maneja unas 3,000 ha de bosque en el que participan los estudiantes por medio del Aprender-Haciendo. Se trabaja en la silvicultura, incluidos la prevención de los incendios con rondas y contrafuegos y el control de plagas forestales. Zamorano cuenta con colecciones forestales en el campus para educación e investigación. El herbario Paul C. Standley tiene más de 300,000 especímenes. El museo de insectos tiene más de 200,000 especímenes que incluye 324 especímenes tipo y especímenes de al menos 14 especies endémicas de Honduras. A esta colección se está integrando una donación de 250,000 especímenes del Dr. Jean Michel Maes de Nicaragua y será una de las más grandes de Centroamérica. Las colecciones del herbario y del museo de insectos fueron la base para la creación del Centro Zamorano de Biodiversidad dedicado al fomento del conocimiento y la conservación de la biodiversidad. Otros centros de excelencia académica tiene Zamorano para mejorar el ambiente, el Centro de Certificación de Estufas Mejoradas y el Centro Zamorano de Energía Renovable. También, Zamorano desarrolla los programas de Eco-Zamorano y Zamorano Verde como estrategias para la protección ambiental y eficiencia de su matriz energética.

Palabras clave: Agua, biodiversidad, energía, forestales, herbario, insectos, producción más limpia.

\section{Zamorano Environmental roll: Environment and Biodiversity Protection}

\begin{abstract}
Zamorano is a university dedicated to forming professionals in the agricultural sciences. Many social and environmental responsibilities are integral parts of this formation. An example of this responsibility is that the university offers formal training in environmental engineering and development thru the Department of Environment and Development. During its 70 years of existence, Zamorano has continually increased its activities in favor of the environment. By 1948 Zamorano's first director, Dr. Wilson Popenoe, proposed the creation of a biological reserve for protection of the Uyuca cloud forest. These management activities have culminated nowadays with the highest efficiency of any protected area in Honduras. The Uyuca Biological Reserve provides ecosystem services of great value, especially potable water for our community. Zamorano manages nearly 3000 ha of forest in which students take part by means of the learning by doing training program. The tasks include aspects of forestry, including fire prevention and control measures by clearing brush and controlled burns, and the control of insect pests. Zamorano possesses several forest species collections on the campus, useful for education and research purposes. The Paul C. Stanley herbarium contains more than 300,000 specimens. The Zamorano insect collection has more than 200,000 identified specimens, including 324 type specimens and 14 species endemic to Honduras. A donation of 250,000 insect specimens organized by Dr. Jean Michel Maes from Nicaragua is being integrated to the collection, and will be one of the largest in Central America. The herbarium and the insect collections were the basis for creating the Zamorano Biodiversity Center dedicated to the promotion of generating new knowledge and conservation of the biodiversity. Other centers of academic excellence operate in Zamorano for environmental improvement. These include the Center of Certification of Improved Stoves and the Zamorano Center for Renewable Energy. At the same time Zamorano is developing Eco-Zamorano and Green Zamorano programs as strategies for better environmental protection and improved efficiency in energy utilization.
\end{abstract}

Key words: Biodiversity, clean production, energy, forestry, herbarium, insects, water, watersheds.

\footnotetext{
${ }^{1}$ Centro Zamorano de Biodiversidad, Departamento de Ambiente y Desarrollo, Escuela Agrícola Panamericana, Zamorano, Honduras, correo electrónico jmora@zamorano.edu

${ }^{2}$ Consultora Ambiental, ecología acuática y macroinvertebrados acuáticos, luciaisa2@ gmail.com
} 


\section{Introducción}

La Escuela Agrícola Panamericana, establecida en 1942 en el Valle del Yeguare en Honduras, se ha enfocado en la formación de profesionales en las ciencias agrícolas. Una producción agrícola más eficiente significa una contribución directa a la conservación de los recursos naturales. En este sentido, desde sus inicios, Zamorano incluyó temas de relevancia en el cuidado del ambiente tales como el agua y el suelo. Actualmente, se dictan cursos específicamente en la conservación de estos recursos imprescindibles para la producción agrícola.

Zamorano ha ido incrementando paulatinamente otros aspectos relacionados al mejoramiento ambiental y la protección de los recursos naturales. Durante su desarrollo se estableció un departamento de recursos naturales y en la actualidad existe el Departamento de Ambiente y Desarrollo que dicta la carrera de Ingeniería en Ambiente y Desarrollo. Al mismo tiempo, se ha trabajado fuertemente en la investigación aplicada en programas como el manejo integrado de plagas, producción y mejoramiento de semillas, y otros que tienen una implicación y relevancia directa con el mejoramiento ambiental y la protección de los recursos naturales.

Zamorano ha incluido en sus actividades diarias el conocimiento de tres pilares fundamentales de la agricultura y la biodiversidad: la biodiversidad doméstica, las plantas y los insectos. La biodiversidad doméstica es la base de todas las sociedades humanas ya que todas las especies que utilizamos provienen de la biodiversidad silvestre. El trabajo con múltiples especies y variedades agrícolas y ganaderas ha propiciado y formado parte del conocimiento de la biodiversidad doméstica de cientos de profesionales de Latinoamérica y otras áreas.

Dos de las situaciones ligadas al trabajo con la biodiversidad doméstica son las plantas y los animales que perjudican a los sistemas agropecuarios y sus especies o variedades. Como resultado, Zamorano estableció dos unidades de suma importancia en su labor de la enseñanza agrícola, un herbario y un museo de insectos. Hay varios otros aspectos relacionados con el estudio y el mantenimiento de la biodiversidad doméstica tales como la investigación con granos y semillas, el control biológico de plagas y varios otros que no son el objeto principal de este trabajo.

Las colecciones de plantas, de insectos y de forestales de Zamorano fueron las bases para la creación de un centro de biodiversidad. Con ello, Zamorano entró oficialmente en este campo de los recursos naturales. No obstante, la conservación de la biodiversidad ha sido un tema clave en el pensum de la Carrera de Ambiente y Desarrollo de Zamorano desde hace varios años. El centro de biodiversidad nació también para administrar un instituto de biodiversidad regional recién creado y cuya sede fue adjudicada a Zamorano por cinco años.

Otros de los temas básicos son el manejo ambiental, la producción más limpia y la energía renovable. Zamorano dicta cursos en estas áreas como parte de su pensum académico. También desarrolla el programa Eco-Zamorano, dedicado a varios aspectos claves para la protección ambiental como el manejo de los desechos sólidos y líquidos, el reciclaje y la racionalización del agua y la energía. Además, cuenta con su programa de Zamorano Verde como una visión amplia del plano ambiental que incluye el ahorro energético y varios otros aspectos de un estilo de vida institucional acorde con la conservación ambiental.

Zamorano realiza múltiples actividades en pro del ambiente y al mismo tiempo participa en eventos relacionados a la conservación ambiental y de los recursos naturales. Dentro de estos destaca la Semana del Ambiente que celebra Zamorano cada año alrededor del 5 de junio, día del ambiente en el mundo. Estas actividades tienen como finalidad principal el aumento de la conciencia ambiental dentro de la institución. Dentro de las actividades que se han efectuado resaltan las de reforestación dentro de los terrenos de Zamorano y en las comunidades cercanas.

Otro aspecto ambiental y de fuerte impacto en la protección de los recursos naturales es el manejo de cuencas. Este tema ha sido uno de los pilares fundamentales de la proyección local en los últimos 18 años en Zamorano. Varios proyectos han sido ejecutados que incluyen la proyección y también programas de capacitación. También la investigación ha sido de importancia clave en el trabajo de cuencas en Zamorano (Caballero B. 2011). 


\section{Biodiversidad}

El término biodiversidad es una contracción de diversidad biológica y como tal fue acuñado por Walter G. Rosen en 1985 en un documento preparatorio para el que acabó por denominarse Foro Nacional de Biodiversidad, que tuvo lugar en Washington D.C., Estados Unidos, en septiembre de 1986 (Izco 2004). El término es de gran aceptación, aunque no deja de ser controversial en algún sentido, pero se convirtió en parte vital de la conservación biológica a partir de las ideas de varios biólogos de la conservación, entre los cuales sobresale Edward O. Wilson y su libro en el tema (Wilson 1988). Norse et al. (1986) ampliaron su uso para referirse a la diversidad biológica en tres niveles: genético (dentro de especies), especies (número de especies) y diversidad ecológica (comunidad).

La década de los 90 fue fundamental para establecer una serie de principios acerca de la biodiversidad y su conservación impulsadas principalmente de forma directa o indirecta por la biología de la conservación y sus postulados (Soulé 1985). En esa época se establecieron programas, institutos y convenios de gran relevancia para la biodiversidad mundial y su conservación. En 1992 se adoptó el Convenio sobre la Diversidad Biológica (CBD por sus siglas en inglés), e incluyó entre los componentes de la biodiversidad, a la variabilidad que existen en los genes dentro de un organismo, a la diversidad de las especies y de los ecosistemas. Uno de los principios básicos de toda esta corriente se centra en la necesidad imperiosa de conocer la biodiversidad biológica del planeta. No obstante, uno de los problemas actuales en esta tarea es la falta de taxónomos capacitados en grupos de organismos específicos (Primack et al. 2001).

Herbario Paul C. Standley: El Herbario Paul C. Standley fue creado en 1943 con la fundación de la Escuela Agrícola Panamericana. Este herbario es uno de los más grandes de Centroamérica y tiene ejemplares de todo Mesoamérica y de otros países con los que tiene nexos universitarios y de intercambios. La colección del Herbario Paul C. Standley tiene cerca de de 300,000 muestras botánicas. Esta colección contiene el mayor número de plantas registradas de
Guatemala y Honduras. También contiene el mayor número de especímenes tipo que cualquier otro herbario mesoamericano.

El Herbario Paul C. Standley cuenta con un área para la colección, espacios internos para preparación de especímenes y otras labores, oficinas y biblioteca. Además, cuenta con un espacio de invernadero para el cultivo de colecciones vivas para estudios taxonómicos y ecológicos. Igualmente, cuenta con un área pequeña donde se mantiene una colección de plantas vivas de clima árido.

Como laboratorio y aporte al desarrollo, el herbario es una valiosa herramienta para conocer las plantas tropicales, varias de las cuales son la materia prima de futuras medicinas y otros usos. De igual manera, las labores de publicación y divulgación del herbario son primordiales para la conservación de los bosques mesoamericanos. Varias especies nuevas para la ciencia han sido descritas en publicaciones formales por personal del Herbario Paul C. Standley donde destacan siete especies de árboles del género Dalbergia (Linares y Sousa 2007).

Desde el punto de vista de investigación aplicada, el herbario contiene una alta colección de especímenes de malezas. De igual manera varias publicaciones en este rubro (Pitty 1997, Pitty y Molina 1998), promueven el conocimiento de la biodiversidad de este componente. Además, el Herbario Paul C. Standley juega un papel importante en el Programa de Aprender-Haciendo ya que es utilizado en el módulo de Monitoreo Ambiental. En este rubro cabe anotar que el Jardín de Malezas de la Carrera de Ingeniería Agronómica fue establecido en el 2004. Es una herramienta para ayudar a los estudiantes en la identificación de las principales malezas en los cultivos en Zamorano. La capacidad máxima del jardin de Malezas de Zamorano es de 150 especies y ya se cuenta con 140 especies.

El Herbario Paul C. Standley desarrolla proyectos de investigación y conservación de la flora mesoamericana. Solo como ejemplo, la "National Science Foundation" y la "University of Minnesota" desarrollan con Zamorano el proyecto "Limits to range expansion of a widespread tropical oak". Este proyecto cuenta con fondos para que los estudiantes de Zamorano investiguen y para el mantenimiento de un experimento de campo a mediano plazo (2009-2014). 
Biodiversidad y manejo forestal. El desarrollo humano ha generado fuertes impactos a la biodiversidad en donde la destrucción o deterioro de los hábitats, la fragmentación de éstos, la sobreexplotación de las especies, la contaminación y la introducción de especies exóticas o extranjeras son las principales causas de la pérdida de la misma (Primack et al. 2001). Al menos un sector de la sociedad humana ha reconocido los valores de la biodiversidad así como los problemas que enfrenta y, lo que es más importante, la necesidad de tomar acciones para su conservación. Es por ello que se necesita trabajar en pro del conocimiento preciso acerca de las causas que determinan la diversidad biológica, su mantenimiento y las herramientas necesarias para detener su pérdida.

Uno de los componentes principales de la biodiversidad regional son las formaciones boscosas pues son el sustrato de una riquísima biodiversidad animal y de otros grupos. A la vez, los bosques son elementos esenciales del desarrollo de la sociedad. Es por ello que el manejo forestal es una de las prioridades de la carrera de IAD, en una región donde varias zonas y países son de vocación forestal. La Unidad Forestal de Zamorano es responsable de la protección y manejo de más de 3,000 ha de bosques de la Universidad. Las áreas de interés incluyen bosques secundarios, bosques maduros y plantaciones en áreas de valle y montaña situados en el cerro Uyuca, Santa Inés y en otros sitios del valle del Yeguare.

Las formaciones forestales son afectadas por varios agentes entre los que destacan los incendios y las plagas forestales. Estos problemas causan los mayores daños ecológicos, económicos y sociales a los bosques de Honduras. Ambos agentes provocan impactos negativos directos al bosque y al ambiente y limitan la provisión de los bienes y servicios ecosistémicos de este ecosistema. Las áreas de bosque manejadas por Zamorano no están exentas a estas amenazas. Debido a ello, la Unidad Forestal de Zamorano elabora y ejecuta planes de protección y vigilancia para dichas áreas. Estos planes son revisados y aprobados por el ICF quién a la vez provee los permisos para la cortas de aprovechamiento o para el control de plagas.

Zamorano invierte mucho dinero para prevenir los incendios forestales por medio de rondas $y$ cortafuegos y en el combate directo de los incendios. A la vez, se trabaja en colaboración con grupos de respuesta organizados de los municipios de San Antonio de Oriente y Tatumbla. El combate de incendios reduce las pérdidas de la cobertura forestal y sus consecuencias asociadas. La reducción de incendios significa una reducción de la contaminación causada por estos. Esta actividad reduce la emisión de dióxido de carbono y otros contaminantes que afectan a la salud pública y contribuyen al efecto invernadero.

Colecciones Forestales de Zamorano. Algunos de los recursos más valiosos para enseñanza en Zamorano son las colecciones botánicas que albergan el herbario (muestras botánicas) y las plantaciones forestales (individuos vivos). Estas últimas contienen una amplia y diversa colección de especies vegetales procedentes de muchas partes del mundo. La mayoría de estas colecciones se encuentra en el campus central y en sus alrededores e incluyen árboles, arbustos, plantas de ornato, frutales, condimentos, plantas medicinales, palmas y malezas. Una colección adicional está constituida por lotes bajo líneas de mejoramiento genético forestal establecidas bajo convenio de manejo con la prestigiosa universidad de Oxford de Inglaterra.

La historia de las colecciones forestales de Zamorano se remonta a los inicios de la institución cuando el Dr. W. Popenoe comenzó a sembrar las primeras especies nativas y exóticas hace ya 70 años. En la actualidad, estas colecciones resguardan un acervo genético de valor inestimable en tiempos cuando la pérdida de este componente de la biodiversidad afecta a miles de especies (Wilson 1988). Se debe reconocer el esfuerzo y empeño del Profesor Nelson Agudelo en el cuidado, creación y manejo de las colecciones forestales de Zamorano. Esta labor está en manos de la Unidad Forestal de Zamorano liderada por el Ing. Timothy Longwell.

En el propio campus central de Zamorano están la Colección de Frutales y Condimentos en la Carrera de Ciencia y Producción Agropecuaria y el Arboreto Simón E. Malo. Solo estas dos colecciones contienen más de 300 especies de plantas pertenecientes a 278 géneros en 56 familias botánicas. Todos los individuos de estas especies, así como las demás colecciones de plantas vivas de Zamorano, necesitan de tratamientos silvícolas adecuadas que requieren la aprobación del 
Instituto Nacional de Conservación y Desarrollo Forestal, Áreas Protegidas y Vida Silvestre (ICF). Estos tratamientos incluyen podas, raleo, eliminación de individuos de especies indeseables o invasoras e incluso el aprovechamiento de algunos individuos que han llegado a su madurez. Los estudiantes de Zamorano participan en estas prácticas silvícolas y adquieren destrezas en el manejo de este tipo de colecciones. Estas actividades aumentan la conciencia ambiental de los futuros profesionales agrícolas de la región.

En el primer semestre de 2011, el ICF expidió la Resolución No. 759 que certifica que las colecciones fueron establecidas por Zamorano y se garantiza al beneficiario el usufructo de los árboles plantados. Las colecciones certificadas ocupan un área de 134 ha, 43 (32\%) de las cuales se encuentran en el campus central. Las otras colecciones están en las áreas periféricas al campus central e incluyen el Arboreto Simón E. Malo, una superficie de 6 ha dedicadas enteramente a las especies forestales que contiene y que constituye un área verde de gran belleza (Figura 1). En esta área verde los estudiantes no solo tienen la oportunidad de esparcimiento sino de práctica y reflexión sobre como su universidad vela por el bienestar humano, la conservación de los recursos naturales y la protección del ambiente.

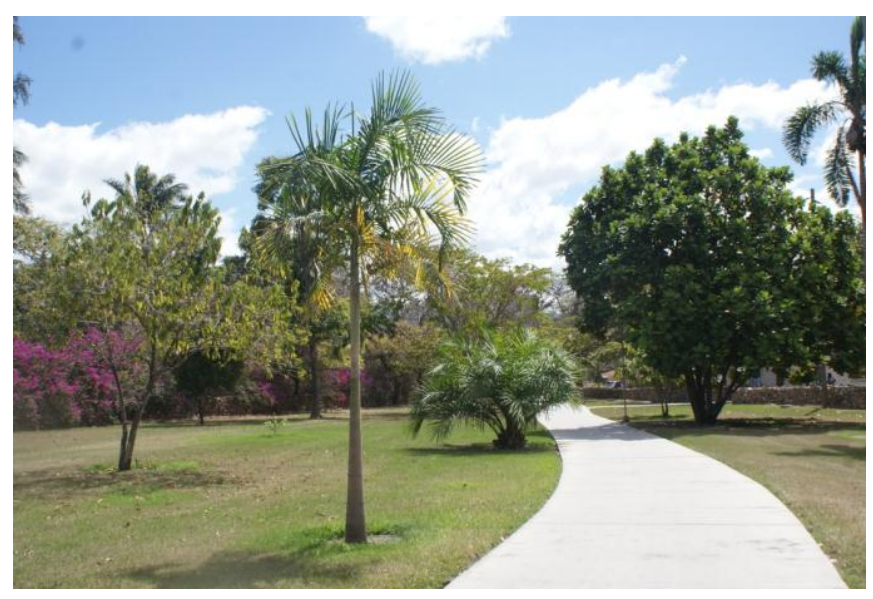

Figura 1. Arboreto Simón E. Malo al costado oeste del campus central de la Escuela Agrícola Panamericana Zamorano (foto J. M. Mora).
El museo de insectos. La Escuela Agrícola Panamericana, como parte de su Centro Zamorano de Biodiversidad (CZB), maneja una de las colecciones de artrópodos más grandes e importantes de la región. La Colección de Artrópodos de Zamorano cuenta con más de 200,000 especímenes de insectos, entre los que destacan 324 especímenes tipo de gran valor científico. La colección incluye larvas de insectos, arácnidos, milpiés, ciempiés y moluscos conservados en alcohol.

La colección incluye especímenes de por lo menos 14 especies endémicas de Honduras, incluidas 10 especies de escarabajos. Destaca la colección de parasitoides, particularmente varias familias de himenópteros y Tachinidae de Diptera. Estos organismos son de gran importancia en el control biológico y el manejo integrado de las plagas. Esto debido a que son enemigos naturales de especies de importancia económica que son plagas en la agricultura. Entre estas plagas destacan los pulgones, las moscas blancas, las escamas, los saltahojas, los picudos y los gorgojos.

La Colección de Artrópodos está incluida en una red de cooperación con organizaciones nacionales, regionales e internacionales. En el marco de esta red se trabaja constantemente en proyectos e investigaciones sobre entomología, especialmente con la biodiversidad de artrópodos, el control biológico y el manejo integrado de las plagas de Centroamérica.

Varios científicos donan muestras a la colección y son depositadas junto con las recolectas del personal del museo. El Dr. Jean-Michel Maes ha donado la colección del Museo Entomológico de León, Nicaragua. Esta donación comprende más de 250,000 especímenes, colectados principalmente en Nicaragua. Como resultado, la Colección de Insectos de Zamorano tendrá casi medio millón de especímenes en el futuro cercano. Ésta será la colección más importante y representativa de Nicaragua y Honduras así como una de las colecciones más grandes de Centroamérica. Existe un convenio oficial sobre esta incorporación científica, la cual fue iniciada en 2008 por gestiones del Centro Zamorano de Biodiversidad.

En cooperación con otros países, Zamorano, a través del Centro Zamorano de Biodiversidad, coordina y apoya proyectos entomológicos que 
implican la recolección de insectos en regiones diferentes de Honduras. Esto aumenta el conocimiento de la biodiversidad nacional y regional lo que resulta en beneficios para su conservación. Varios proyectos se desarrollan en las áreas protegidas de Honduras. Así que el buen cuidado de esta colección científica es una tarea muy importante para obtener y facilitar datos sobre la riqueza biológica de esas áreas protegidas.

La Colección de Artrópodos ofrece un sinnúmero de posibilidades para las prácticas, el AprenderHaciendo, la enseñanza y las tesis de los estudiantes de Zamorano. El módulo de Monitoreo Ambiental del Aprender-Haciendo utiliza constantemente la colección.

El Centro Zamorano de Biodiversidad. En un esfuerzo por reunir las colecciones biológicas de Zamorano para el conocimiento y uso racional de la biodiversidad, se creó el Centro Zamorano de Biodiversidad (CZB) en 2005. Una de las tareas inmediatas a la creación del CZB fue administrar el Instituto Regional de Biodiversidad (IRBIO) de la Comisión Centroamericana de Ambiente y Desarrollo (CCAD). Así, el CZB se convirtió en el brazo técnico de la CCAD en materia de biodiversidad al facilitar la ejecución de proyectos relacionados con el IRBIO. El CZB tiene como finalidad trabajar en pro de la biodiversidad y su conservación mediante el desarrollo de proyectos que contribuyen al desarrollo sostenible.

Varias actividades del CZB han sido al servicio del gobierno de Honduras. Por ejemplo, a petición de la Empresa Nacional de Energía Eléctrica (ENEE) se evaluó la biodiversidad del área forestal protegida de El Cajón en 2010. La importancia de este diagnóstico radicó en la necesidad de satisfacer la demanda de información actualizada y completa sobre los recursos naturales del área. Esta información es esencial para la toma de decisiones conservacionistas, así como para establecer controles y vigilancia más efectivos en el área para reducir las presiones sobre la misma.

En el Valle de Sico se ha trabajado con el ICF en varios componentes relacionados a la conservación ambiental. Este trabajo se ha realizado en colaboración con la fundación Madera Verde y el Proyecto ECOSISTEMAS. Las actividades incluyen la educación ambiental con los talleres Jaguares para Siempre. Para este componente se trabajó en las escuelas primarias de la Moskitia. Además, se realizaron talleres con ganaderos para ayudar a entender la biología de los murciélagos y sobre los métodos para su control. Para esto se trabaja con ganaderos que están participando en actividades referentes a la conservación del jaguar en Sico. También se apoyó la elaboración del Plan Nacional de Conservación del Jaguar (ICF 2011).

El CZB ha apoyado al ICF con la generación de protocolos para el monitoreo biológico de siete Objetos de Monitoreo en Paisajes Productivos. Estos protocolos se ejecutan para el monitoreo de los objetos correspondientes en Sico en Iriona, Departamento de Colón y en Texiguat en el Departamento de El Paraíso. En esta última zona ya se han realizado algunos proyectos de investigación con tesis de estudiantes de la carrera de IAD como apoyo para el establecimiento de un corredor biológico.

El CZB ha participado con ponencias y charlas en diversos foros $y$ congresos internacionales relacionados a la biodiversidad, tales como el VI Congreso Mundial de Herpetología celebrado en Brasil en el 2008 y los congresos de la Sociedad Mesoamericana para la Biología de la Conservación. El centro ha organizado y ejecutado reuniones internacionales en Zamorano con expertos regionales para determinar el estado de conservación de los mamíferos, los anfibios y los ecosistemas críticos de Mesoamérica. Además, los investigadores asociados al CZB han participado en varias publicaciones científicas en libros y revistas científicas internacionales (Gascon et al. 2012, Castañeda y Mora 2010, Espinal et al. 2010, López et al. 2010, Marineros et al. 2012, Mora 2012a, Mora 2012b).

El IRBIO, administrado por mandato por el CZB, ha jugado un papel importante en facilitar la comunicación regional (Centro América y República Dominicana) de los Comités Técnicos nacionales de áreas protegidas, biodiversidad y de sistemas de información ambiental. Esto se ha logrado mediante la organización de reuniones frecuentes para estos grupos. También ha ejecutado varios proyectos en Centro América. Uno de los proyectos más prometedores fue el Programa Estratégico de Monitoreo y Evaluación de la Biodiversidad financiado por el BID. Desafortunadamente, algunas decisiones técnicas y administrativas no permitieron la 
elaboración de algunos protocolos de monitoreo de la biodiversidad en Centro América tal y como se había aprobado. No obstante, aunque el convenio de administración del IRBIO ya caducó, se trabaja en una segunda etapa del PROMEBIO.

Un resultado de gran éxito del IRBIO fue la ejecución de un proyecto de modelación de la biodiversidad en la región. Bajo éste se prepararon y publicaron modelos de distribución de la biodiversidad en Centroamérica así como un diagnóstico del estado actual y futuro de la biodiversidad en Centro América (Triminio y McLean 2011). De igual manera, fue muy exitosa la ejecución del proyecto Utilizando las Pesquerías de Pequeña Escala para Monitorear la Biodiversidad Marina: Aplicación del Índice Trófico Marino como Herramienta de Monitoreo en las Aguas Tri-Nacionales del Golfo del Fonseca, Elaborado por CEMU para CZB/IRBIO (Mora 2012).

Varios otros proyectos se ejecutaron durante el tiempo de administración del IRBIO por el CZB. Destacan aquellos proyectos de aplicación regional al seguir la misión del IRBIO de ser un ente de acción para toda Centro América y la República Dominicana. Entre estos proyectos cabe destacar la preparación de una estrategia para la conservación de especies crítica y globalmente amenazadas en Mesoamérica Norte (Garcia-Moreno et al. 2006). De este taller se generaron otros proyectos como el de los árboles y epífitas de Mesoamérica de gran relevancia para la conservación regional de la biodiversidad.

\section{Otros Centros de Excelencia Académica}

Dos centros de excelencia académica adicionales trabajan en pro del ambiente en Zamorano. Estos son el Centro de Energía Renovable (CZER) y el Centro de Certificación de Estufas Mejoradas (CCEM). El CZER trabaja en la investigación e innovación para enfrentar nuevos retos relacionados al campo energético y la conservación ambiental. El CCEM ayuda a organizaciones no gubernamentales y otros agentes de desarrollo a proveer a la población pobre de Centroamérica con estufas mejoradas o ecoestufas que sean eficientes en el uso del combustible y disminuyan la contaminación ambiental.

El CZER se fundó en 2007 con la misión de promover, adaptar y evaluar las tecnologías de energía renovable para Honduras y América Central. Se ha trabajado en varios proyectos tales como la generación de mercados de energía renovable en la Mancomunidad del Yeguare; biogás de residuos agrícolas para la producción de electricidad; capacitación, investigación y promoción sobre energía renovable en el sector agropecuario y forestal en la región Centroamericana.

Con estos proyectos se pretende capacitar a los técnicos de la zona en tecnologías de energía renovable. Por ejemplo, la preparación en la instalación de sistemas solares fotovoltaicos en escuelas y residencias. Además, ofrecer asesoría técnica en energía renovable a las comunidades aledañas de Zamorano, la creación de oportunidades de financiamiento en alianza con microfinancieras de la zona, la adquisición de equipo de pretratamiento de desechos agrícolas y la adquisición de biodigestores tubulares de geomembrana.

El tema de producción más limpia es también prioritario en Zamorano y se incluye en el pensum académico formal de los estudiantes de la carrera de IAD. En esta temática se han conducido algunas investigaciones en el campus. Recientemente, Zamorano firmó una carta de acuerdo para llevar a cabo una auditoría energética con fondos del BID. La idea es entender el uso de la energía en el campus, las oportunidades existentes para la conservación de la energía y la generación sostenible en el campus. La donación del banco se hace dentro del programa de Incremento de la Inversión en Energía Limpia en Centroamérica.

El Centro de Certificación de Estufas Mejoradas (CCEM) fue creado en 2009 con financiamiento de TPW Energy Collaborative. El CCEM es el único en su clase en América Latina y fue diseñado basado en otros centros de investigación que se encuentran en Asia y África. El centro trabaja en varios frentes para promover las tecnologías eficientes de estufas en Centroamérica. El CCEM evalúa modelos de estufas en términos de eficiencia de uso de la leña, las emisiones de gases tóxicos y su aceptación en las comunidades locales (Figura 2).

Las estufas mejoradas ayudan a combatir la pobreza, mejoran la calidad del aire y la salud pública. Con mayor eficiencia en el uso del combustible, las familias pueden invertir más tiempo en el trabajo y en 
el estudio ya que necesitan menos tiempo para recolectar leña. También economizan ya que gastan menos dinero porque compran menos leña. Esto tiene una connotación ambiental positiva ya que se contribuye a la reducción de la deforestación. A la vez, la disminución de los gases tóxicos incide en una mejor calidad del aire y contribuye a disminuir las enfermedades respiratorias en las familias que utilizan las estufas mejoradas.

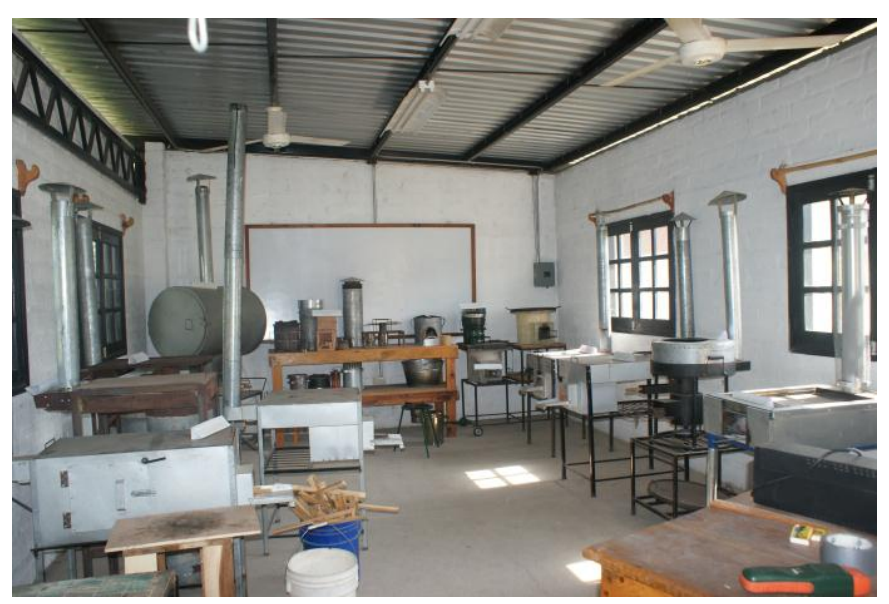

Figura 2. Estufas sometidas a la evaluación de su eficiencia energética y la emanación de gases tóxicos en el Centro de Certificación de Estufas Mejoradas de Zamorano (foto J.M. Mora).

Aunque la mejora en la eficacia en el uso de estufas ha sido promovida durante los últimos 35 años, todavía hay que evaluar el impacto de estos esfuerzos en Centroamérica. No existe una organización que regule o controle la calidad de la construcción de la estufas o que certifique que su funcionamiento cumple con los objetivos esperados. Por lo tanto, hay que estandarizar los materiales de construcción, las especificaciones del diseño y el desempeño ambiental de los modelos de estufas para asegurar y cuantificar la reducción del impacto ambiental al remplazar las estufas tradicionales por estufas mejoradas. El CCEM tiene un laboratorio de certificación, equipo para medir la eficiencia del consumo de leña y 15 modelos de estufas mejoradas comunes en América Latina para evaluación y enseñanza.

\section{Eco-Zamorano}

Ya en los años 1940, en los inicios de Zamorano, se dieron las primeras acciones de gestión ambiental en Zamorano por parte del Dr. Wilson Popenoe y sus asesores. Ellos diseñaron las lagunas de estabilización de Zamorano lo que dio inicio al exitoso proyecto para el tratamiento de las aguas residuales que a la vez conservó la belleza escénica natural del entorno (Quiroz 2013). El siguiente resumen está basado en Quiroz (2013) y detalla algunos aspectos relevantes del programa Eco-Zamorano.

A principios de la década del 2000, Zamorano impulsó varias actividades orientadas a mejorar la gestión de los residuos sólidos y las aguas residuales en el campus. Se hizo un diagnóstico situacional del manejo de los residuos sólidos, la selección técnica ambiental de un sitio para el relleno sanitario y la optimización de las lagunas de estabilización para el reciclaje de las aguas depuradas en el sector agrícola. Tales proyectos motivaron a las autoridades a crear un programa de manejo ambiental, al que se llamó EcoZamorano, con el objetivo de brindar el seguimiento debido a estos proyectos.

Eco-Zamorano resultó en exitosos proyectos planificados, diseñados e implementados por el programa con el apoyo de la Gerencia de Mantenimiento entre el 2004 y 2010. Entre estos proyectos destaca el primer manejo integral de los residuos sólidos implementado en Honduras. Este proyecto incluye la recuperación de los materiales reciclables a través de centros de acopio localizados en todo el campus. En este periodo se construyó el primer relleno sanitario en Honduras con el tratamiento de líquidos lixiviados con tecnologías apropiadas. Se implementó el manejo de los residuos peligrosos procedentes de las actividades agrícolas, los laboratorios, las oficinas y las plantas de producción. Además, se implementó el compostaje de los residuos orgánicos biodegradables producidos en el campus, en coordinación con el Departamento de Ciencia y Producción Agropecuaria. Este compostaje reduce el consumo de fertilizantes sintéticos.

Eco-Zamorano elaboró en el 2005 el primer plan de racionalización del agua y la energía. Éste incluyó diversos proyectos como la implementación de biodigestores en las plantas de ganado lechero y 
porcino así como en las lagunas de estabilización, la sustitución de las calderas de combustible fósil por biomasa, los pagos por servicios ambientales tales como la tarifa por el consumo de agua, la instalación de sistemas de iluminación más eficientes, la optimización del sistema de identificación de fugas en la red de agua potable, los estudios hidrogeológicos, el incremento en el reciclaje de las aguas residuales para el riego de cultivos y pastos, la recuperación de las aguas lluvias en las edificaciones, los calentadores solares, proyectos eólicos, proyectos microhidroeléctricos en las redes principales de agua tales como Uyuca y Santa Inés y una central hidroeléctrica en el río Yeguare.

Los proyectos de Eco-Zamorano motivaron la participación de los estudiantes de los departamentos de Ambiente y Desarrollo y Ciencia y Producción Agropecuaria a través de la elaboración de sus proyectos especiales de graduación en las unidades de ganado porcino y lechero y en las lagunas de estabilización. Se instaló un primer biodigestor en la unidad de ganado lechero en el 2009. Después de eso se han instalado biodigestores en las lagunas de estabilización y en la unidad de ganado porcino.

Como resultado del plan de racionalización del agua y la energía, la Gerencia de Mantenimiento procedió a la instalación de sistemas más eficientes de iluminación así como el primer calentador solar de agua en una residencia del campus central. Además, Eco-Zamorano desarrolló en el 2007 un estudio de prefactibilidad para la implementación de una pequeña central hidroeléctrica. También se ejecutó el primer estudio hidrogeológico en un sector del campus central, que resultó en la construcción del pozo que abastece la demanda de riego del campo de fútbol Tony Smith.

Durante la implementación de Eco-Zamorano, los departamentos de Agroindustria Alimentaria y Ciencia y Producción Agropecuaria desarrollaron proyectos que contemplan el manejo ambiental de sus operaciones. Estos proyectos son la construcción de trampas de grasa en las plantas de lácteos y cárnicos y el compostaje de residuos en la unidad de aves.

Otro aspecto relevante en el manejo ambiental de Zamorano, fue la gestión de las licencias ambientales a través de las oficinas de Asesoría Legal y EcoZamorano en los proyectos que se desarrollaron a partir del 2004. Sin embargo, Zamorano carecía de una licencia ambiental para todas las operaciones anteriores a ese año. Debido a ello, Eco-Zamorano y Asesoría Legal lideraron una auditoría ambiental de toda la universidad en el 2009, que resultó en la adquisición de la licencia ambiental en el 2012.

Zamorano genera 4,744 $\mathrm{kg}$ diarios de residuos sólidos y su producción per cápita es de $0.70 \mathrm{~kg}$. A través de un manejo integral de residuos sólidos, $13 \%$ es recuperado para su comercialización como material reciclable tales como envases, papel, latas, vidrio y chatarra. Además, $67 \%$ de los residuos es aprovechado para la producción de abono orgánico y de esta forma se reduce el consumo de fertilizantes sintéticos en los cultivos agrícolas.

Los recipientes plásticos vacíos de agroquímicos son tratados con un triple lavado y posteriormente incinerados a temperaturas superiores a $800{ }^{\circ} \mathrm{C}$ en una compañía cementera. El resto de recipientes de agroquímicos son depositados en una estructura hermética para prevenir su contacto con el agua de lluvia y la contaminación de los recursos hídricos (Quiroz 2013).

Únicamente $20 \%$ de los residuos sólidos generados en Zamorano son depositados en su propio relleno sanitario. La recuperación de materiales reciclables y peligrosos permite incrementar la vida útil del relleno y reducir la contaminación del suelo. El relleno cuenta con chimeneas para evacuar los escasos gases inflamables generados debido a que su principal fuente constituida por materiales biodegradables es recuperada para la producción de abono orgánico. Finalmente, el sitio cuenta con un sistema de drenaje para evacuar los líquidos lixiviados y trasladarlos a un tratamiento pasivo.

El tratamiento pasivo en el relleno consiste en una laguna de evaporación y estabilización del lixiviado donde $52 \%$ de la materia orgánica biodegradable es depurada. Además, se depura $46 \%$ de la materia orgánica no biodegradable, $28 \%$ de los coliformes fecales, $40 \%$ del nitrógeno, $33 \%$ de los sólidos y $68 \%$ de los aceites y las grasas. El tratamiento es completado por la evaporación del lixiviado durante la estación seca debido a su diseño que permite el almacenamiento durante la estación lluviosa. 


\section{Zamorano Verde}

En el 2009 surgió la iniciativa de crear una imagen institucional que integraría todos los esfuerzos en el contexto ambiental en Zamorano. En el 2012 se ha reimpulsado esta iniciativa enmarcada en el subobjetivo cinco del objetivo estratégico tres del plan estratégico de Zamorano. Zamorano Verde pretende integrar no solo los proyectos de carácter ambiental en las instalaciones físicas y la infraestructura, sino también a toda la comunidad Zamorana, sean estos empleados residentes o no residentes, así como a la comunidad estudiantil (Quiroz 2013).

El subobjetivo cinco del objetivo estratégico tres pretende fortalecer el programa Zamorano Verde para desarrollar y aplicar una cultura que incluya el uso responsable de la energía renovable, el agua, los recursos naturales y el manejo de los residuos y las emisiones (Quiroz 2013). Por lo tanto, en este subobjetivo se contemplaron las siguientes actividades:

- Reactivar la estrategia de Zamorano Verde.

- Establecer los indicadores de desempeño de Zamorano Verde tales como agua, residuos, biodiversidad y energía.

- Mejorar los índices de reciclaje y generación de residuos en el campus.

- Diversificar la matriz energética hacia energías limpias y eficientes.

- Implementar la matriz energética basada en el potencial de los recursos con que cuenta la institución.

La base de Zamorano Verde es una campaña institucional para aumentar la eficiencia energética a través del uso de varias fuentes de energía como el biodiesel, el biogás, la hidroeléctrica, la solar y la eólica. La base de esta campaña es que estos tipos de energía se evalúan como parte de la educación y del Aprender-Haciendo de Zamorano, lo que convierte a la institución en un laboratorio vivo. Sin embargo, el nuevo plan estratégico de Zamorano Verde es más amplio e incluye cinco ámbitos definidos como recursos hídricos, residuos sólidos, residuos líquidos, sensibilización ambiental y energía (Quiroz 2013).

El Departamento de Ambiente y Desarrollo creó el Centro Zamorano de Energía Renovable en el 2009. Este centro ha aportado a la gestión de fondos para el impulso de los proyectos en los departamentos de Zamorano. Además, el Departamento de Ambiente y Desarrollo ha impulsado diversos proyectos como el de los biodigestores y los ecofogones más eficientes para el sector rural (Quiroz 2013).

El Departamento de Ciencia y Producción Agropecuaria inició investigaciones sobre el cultivo de Jatropha curcas y el uso de biodiesel en la maquinaria agrícola. El Departamento de Agroindustria Alimentaria promovió la implementación de una planta de producción de biodiesel y desarrolló varios proyectos especiales de graduación en este tema. El Departamento de Agronegocios ha incursionado en los análisis financieros de los proyectos afines como los calentadores solares en el sector residencial y los proyectos microhidroeléctricos (Quiroz 2013).

Con Zamorano Verde, se han ejecutado varios proyectos relacionados al manejo sostenible de los recursos naturales. Un ejemplo es el proyecto Generación de Mercados de Energía Renovable en la Mancomunidad del Yegüare desarrollado por el Centro Zamorano de Energía Renovable, que formó a 25 microempresarios de energías renovables decididos a contribuir al desarrollo de sus familias y sus comunidades (Zamorano 2011).

\section{La Reserva Biológica del Cerro Uyuca}

Una de las mayores contribuciones ambientales de Zamorano es sin duda la creación, mantenimiento y protección de la reserva biológica del cerro Uyuca (Mora B. y López U. 2011). Esta área protegida, aunque pequeña, provee bienes y servicios ecosistémicos de gran valor ambiental.

Un ambiente sano está dado por los servicios ecosistémicos que se derivan de la existencia de la biodiversidad. Los componentes de la biodiversidad juegan papeles ecológicos definidos que en conjunto dan a su ecosistema el equilibrio necesario para su funcionamiento. Esta función se traduce en bienes y servicios ambientales, más correctamente denominados servicios ecosistémicos, que permiten la subsistencia de las comunidades humanas ya que les son imprescindibles.

Los principales servicios ecosistémicos de Uyuca son el aire puro mediante la absorción de dióxido de carbono y producción de oxígeno; agua, por medio de 
la retención de las lluvias en la vegetación y en el suelo y la captación de lluvia horizontal; suelos, por medio de su producción y fertilidad por la descomposición vegetal y la retención ante la escorrentía. Otros servicios ecosistémicos son menos tangibles como la descomposición de la materia y los ciclos biogeoquímicos en donde participan organismos que incluye principalmente bacterias y hongos.

Consciente de esta situación, Zamorano ha incluido en su nuevo plan estratégico acciones para mantener los servicios ecosistémicos que se derivan de las tierras que maneja. Dentro de estas tierras destaca la Reserva Biológica del Cerro Uyuca (Figura 3). Casi todas las montañas alrededor del valle del Yeguare han sido muy afectadas por la intervención humana, lo que nos ha desprovisto de sus servicios ecosistémicos. Esos servicios se han perdido porque se han perdido los elementos de la biodiversidad que los proveen. Pero no solo se ha alterado el ecosistema, sino que se han perdido especies, genes $\mathrm{y}$ asociaciones $\mathrm{y}$ fenómenos ecológicos fundamentales. Por todo ello, Uyuca se convierte en uno de los últimos reductos para albergar muchas especies vegetales, animales $\mathrm{y}$ otros organismos.

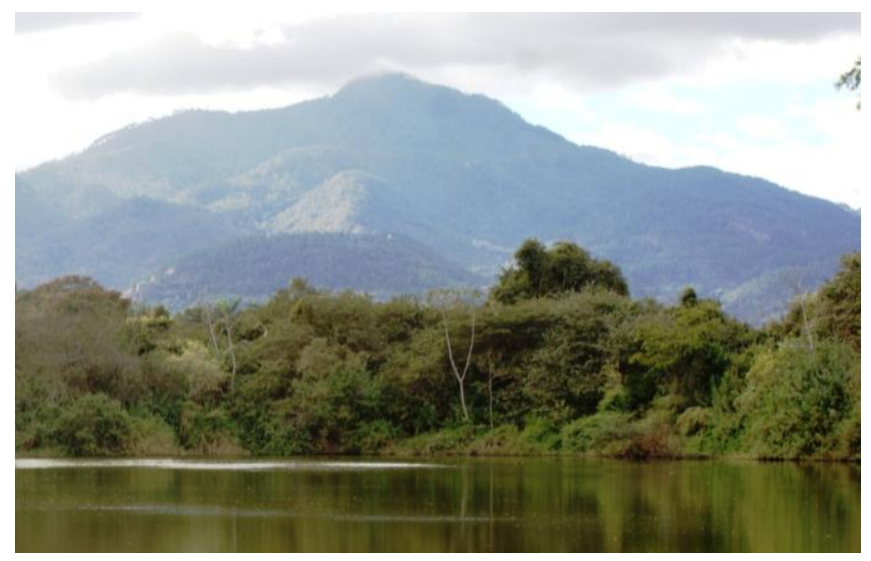

Figura 3. Vista del cerro Uyuca en donde se encuentra la Reserva Biológica Uyuca, visto desde una de las lagunas de estabilización que tiene Zamorano para el tratamiento de las aguas servidas (foto J.M. Mora).

Zamorano y Honduras cuentan con la reserva biológica de Uyuca para el estudio científico y el monitoreo ambiental en una región donde ya se han perdido otros sitios que pudieron cumplir estos papeles. Pero aun más importante es que todos los vecinos de Uyuca pueden seguir disfrutando del aire puro, agua abundante, suelos fértiles y biodiversidad así como la belleza escénica que Uyuca provee.

Durante los últimos dos años, Zamorano ha trabajado en un nuevo plan de manejo para la RBU. Este trabajo se ha estado realizando en coordinación con el ICF y otros actores clave como las municipalidades de San Antonio de Oriente y Tatumbla y las Fuerzas Armadas de Honduras. Se trabaja para oficializar el plan de manejo de la RBU acorde a su misión de reserva biológica (Mora B. y López U. 2011).

Agradecimientos. A todas las personas que proveyeron datos, informes e información acerca de las unidades, proyectos y acciones de Zamorano que se han mencionado en este trabajo. En particular al Profesor Nelson Agudelo quién contribuyó de múltiples formas al mejoramiento ambiental por más de 30 años durante su trabajo en Zamorano; Carlos Quiroz, Victoria Cortés, Jorge Iván Restrepo y Oliver Schlein. Varias porciones de texto fueron tomadas y adaptadas de la página web de Zamorano, particularmente aquellas relativas a las unidades de Zamorano incluidas en el texto.

\section{Literatura Citada}

Caballero B., L.A. 2011. Contribuciones de Zamorano a la Investigación y Proyección en el Manejo de Cuencas en Centro América. Ceiba 51(1):122-141.

Castañeda. F. y J.M. Mora. 2010. Impact of fire on a wetland population of the scorpion mud turtle (Kinosternon scorpioides) in northwestern Costa Rica. p. 706-715. In Wilson, L.D., J.H. Townsend y J.D. Johnson. Conservation of Mesoamerican Amphibians and Reptiles. Eagle Mountain Publishing, LC, Eagle Mountain, Utah.

Espinal. M., J.M. Mora y F. Leiva. 2010. Abundance and distribution of the American crocodile (crocodylus acutus) at El Cajón reservoir, Honduras, and the development of an integrated management plan for conservation. p. 734-745. In Wilson, L.D., J.H. Townsend y J.D. Johnson. Conservation of Mesoamerican Amphibians and Reptiles. Eagle Mountain Publishing, LC, Eagle Mountain, Utah. 
García-Moreno, J., J.I. Restrepo, S. Zabala y S. Triminio. 2006. Memoria del taller "Preparación de una estrategia para la conservación de especies crítica y globalmente amenazadas en Mesoamérica Norte". Zamorano, Honduras. Zamorano, Conservation International, Critical Ecosystem Patnership Fund. 31 p.

Gascon, C., J.P. Collins, D.R. Church, R.D. Moore, F. Andreone, P. Bishop, S.D. Biju, F. Bolaños, X. Feng, L. Pipeng, L. Zhang, H. Shi, S. Lötters, Y. Matamoros, M. Meegaskumbura, S. Molur, P.N. Mitchel, J.M. Mora-Benavides, J. Garcia-Moreno, H. Randriamahazo, J.T. Reardon, C. Molina, S. Ron, J.J.L. Rowley, D. Silvano, P.H. Valdujo y V.K. Verdade. 2012. Scaling a global plan into regional strategies for amphibian conservation. Alytes 29(1-4): $15-27$.

ICF. 2011. Plan Nacional para la Conservación del Jaguar (Panthera onca); "Promoviendo la convivencia Comunidad-Jaguar" Departamento de Vida Silvestre/Instituto Nacional de Conservación y Desarrollo Forestal, Áreas protegidas y Vida SilvestreProyecto Ecosistemas-Fundación Panthera. Tegucigalpa. $29 \mathrm{p}$.

Izco, J. 2004. Biodiversidad y conservación, p. 663-713 en Botánica. J. Izco, coordinador. McGraw Hill, Madrid. $906 \mathrm{p}$.

Linares, J.L. y Sousa, S. 2007. Nuevas especies de Dalbergia (Leguminosae: Papilionoideae: Dalbergieae) en México y Centroamérica. Ceiba 48(1-2):61-82

López, L.I., P. Gutiérrez y J.M. Mora. 2010. Macrofauna Acuática de la Quebrada Santa Inés, Subcuenca del Río Yeguare, Honduras. Ceiba 51(1):17-28

Marineros, L., J. Porras, M. Espinal, J.M. Mora y L. ValdésOrellana. 2012. Manual de reconocimiento de las serpientes venenosas de Honduras. Heliconia Ideas y Publicaciones. $79 \mathrm{p}$.

Mora B., J.M. y López U. 2011. El Manejo de la Reserva Biológica Uyuca en el Contexto Nacional y Global del Sistema de Áreas Protegidas. Ceiba 51(1):39-54.

Mora, J.M. 2010. Natural history of the black spiny-tailed iguana (Ctenosaura similis) at parque nacional Palo Verde, Costa Rica, with comments on the conservation of the genus Ctenosaura. p. 716-733. In Wilson, L.D., J.H. Townsend y J.D. Johnson. Conservation of Mesoamerican Amphibians and Reptiles. Eagle Mountain Publishing, LC, Eagle Mountain, Utah.
Mora, J.M. (Comp.). 2012a. Proyecto Utilizando las Pesquerías de Pequeña Escala para Monitorear la Biodiversidad Marina: Aplicación del Índice Trófico Marino como Herramienta de Monitoreo en las Aguas Tri-Nacionales del Golfo del Fonseca. Elaborado por CEMU para CZB/IRBIO. 47 p.

Mora, J.M. 2012b. Lasiurus egregius (Vespertilionidae) in Honduras, Central America. The Southwestern Naturalist 57(1):104-105.

Norse, E.A., K.L. Rosenbaum, D.S. Wilcove, B.A. Wilcox, W.H. Romme, D.W. Johnston y M.L. Stout. 1986. Conserving biological diversity in our national forest. The Wilderness Society, Washington, D.C. 116 p.

Pitty A., y A. Molina R. 1998. Guía fotográfica para la identificación de malezas: parte II. Zamorano Academic Press, Zamorano, Honduras. 136 p.

Pitty, A. (ed). 1997. Introducción a la Biología, Ecología y Manejo de Malezas. Zamorano Academic Press, Honduras. 300 p.

Primack, R., P. Rozzi, R; P. Feinsinger, R. Dirzo, F. Massardo. 2001. Fundamentos de conservación biológica. México DF. Fondo de Cultura Económica. 797 p.

Quiroz, C. 2013. Zamorano Verde, plan Estratégico 20132018: borrador del plan. Departamento de Ambiente y Desarrollo, Zamorano. 41 p.

Soulé, M. 1985. What is conservation biology? BioScience 35: $727-734$

Triminio, S. y D. McLean. (Comp.). 2011. Estado actual y futuro de la biodiversidad en Centro América/Current and future status of biodiversity in Central America. Reporte técnico/Technical report, Programa Estratégico de Monitoreo y Evaluación de la Biodiversidad (PROMEBIO). Netherlands Environmental Assessment Agency, Convention on Biological Diversity, Zamorano, BID. 216 p.

Wilson, E.O. (ed.). 1988. Biodiversity. National Academy of Sciences/Smithsonian Institution. 538 p.

Zamorano. 2011. Informe anual 2011. EAP Zamorano, Valle del Yeguare. 45 p.

Sometido para publicación el 20 de marzo del 2013. Aceptado para publicación el 27 de abril de 2013. 